\title{
Evidence Aggregation Based Spam Detection in e-Commerce Social Network
}

\author{
Lavanya MP*, Nandini BM \\ Department of Information Science \& Engineering and PG studies, NIE, Mysuru, India. \\ DOI: https://doi.org/10.21467/proceedings.1.70 \\ * Corresponding author email: lavanyampparamesh@gmail.com
}

\begin{abstract}
Ranking spam in the Social Media and Social Network market refers to fake or deceptive activities which have a purpose of striking up the products and the services for different interests in the popularity list. Indeed, it becomes more and more repeated for Social Networks to use sheltered means, such as inflating their products sales or posting services of the product ratings, to commit ranking spam. While the importance of preventing ranking spamming has been recognized, we provide a holistic inspection of ranking spam and propose a spamming detection system for social network. We propose to exactly locate the ranking spam by mining the active periods, namely leading sessions, of social network. Such sessions can be influenced for detecting the actual rating instead of spammed rating of product rankings. by modeling social networks ranking, rating and review behaviors in the course of statistical proposition tests.
\end{abstract}

Index Terms- Ranking Fraud, Spammer, Evidence-Aggregation, Spamming detection.

\section{INTRODUCTION}

The numeral of e-commerce for more information has extend at stunning speed over the gone by few years, humans are put down analysis their decision-making processes, and favorable or nonfavourable analysis in their choice of item and aid in social networking. These evaluation have get in main part in successfulness of a career time, positive reviews can useful for a company, negative reviews can slap and cause economic losses. The reality that anyone with any recognisation can quit statements as reviews and gives a attractive good time for spammers to writing fraud reviews planed to fool users judgement. These deceptive reviews are grow by the split task of social medias. And extend over the network. The reviews note down to alter users' recognition of how better a product or service are appraise as spam, our watchful inspection disclose that e-commerce community websites are not every time rate highest in the leader board, but only in some leading gathering, which configuration dissimilar leading sessions.

(C) 2018 Copyright held by the author(s). Published by AIJR Publisher in Proceedings of the $3^{\text {rd }}$ National Conference on Image Processing, Computing, Communication, Networking and Data Analytics (NCICCNDA 2018), April 28, 2018.

This is an open access article under Creative Commons Attribution-NonCommercial 4.0 International (CC BY-NC 4.0) license, which permits any non-commercial use, distribution, adaptation, and reproduction in any medium, as long as the

AijR license, which permits any non-commercial use, distribution, 


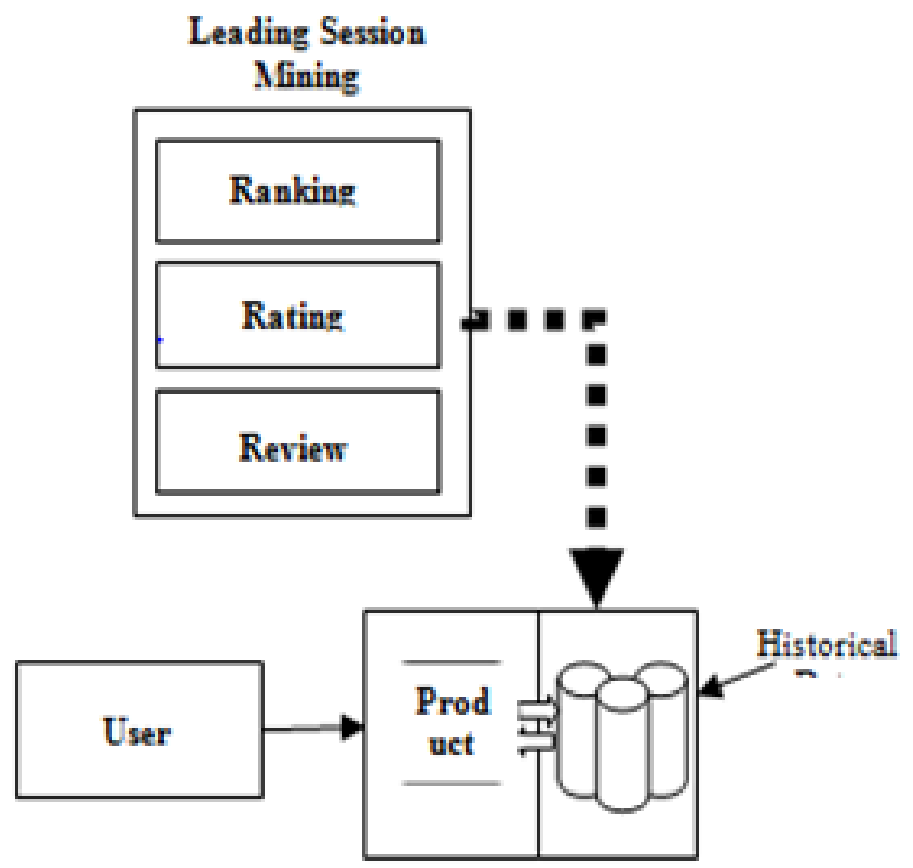

Fig 1: Architecture of netspam detection system for social network

Note that we will start both leading events and leading sessions in specific subsequent. Similarly, ranking fraud normally Appeal in these leading session. On the other side, a significant quantity of literary text has been produce on the capability to recognize spam and spammers as well as disparate kind of survey on this topic. These techniques can be categories into linguistic design in texts which are founded on bigram and unigram, others are based on behavioral decoration that turn on characteristic bring out from design in users' actions which are metadata based and some skills to utilize diagram and chart-form process. The detecting spamming in social network is actually become aware of ranking deception inside leading sessions of social websites. We initial suggest a process to recognize the leading sessions of each item based on its historical ranking records. At the time, with the survey of manufactured item ranking behaviors, we observe that the fake products frequently have unlike ranking devices in each leading session as contrast with common commodity. And express some fraud evidence from product historical ranking records, and develop three functions to extract such ranking based fraud evidences. The ranking based evidences can be affects by e-commerce developers' reputation and some legal marketing, such as "limited-time discount". As a result, it is not enough to use ranking based proves. Therefore, we propose two types of illegal events based on product rating and review history, which reflect some patterns from products historical rating and review records. We develop an evidence-aggregation method to integrate these three types of evidences for evaluating the reliability of leading sessions from social ecommerce media. 


\section{LITERATURE SURVEY}

Many are done in the field of preventing Net Spam attacks by implementing evidence aggregation. Usually preference aggregation issue, in which the various in place of over thing should be adding into a common opinion ranking. Rather than over product can be expressed in a variety of forms, which makes the aggregation problem difficult. In this work M. N. Volkovs et al [1] express an easily modifying the based on a representation over the pair wise correlation that can provides all these forms. Inference in the model is very fast, it making the Application to problems with hundreds of preferences. Experiments on standard datasets show the higher performing a task to existing methods of working.

K. Shi together with K. Ali was suggested the Netflix competition of 2006 [2] had helped significant activity in the awards field, especially in Approaches using covered up factor models the well-known nature of the Netflix and the similar Movie Lens datasets may be reduces the detail of the period of instruction to be well informed in this field. At GetJar, their aim is to prepare the interesting for the mention of web Application. For the usage of purpose, they did see a issuing that has more kurtosis than for the previously was mentioned the feature datasets. It takes place mostly things is available because of the huge of unplanned to Application developers and low cost of Application publication connected to features.

Ntoulas et al [3]. Continue their systematic study of "web spam": we have to vaccination of the fake generated pages into the web in order to affect their results from the search engines, to the drive traffic to certain pages for the profit. In this study author considered as the amount of earlier too unusual techniques for the computerized notice have to spam pages, survey of the effectiveness of these techniques in isolation and when using classification algorithms aggregated. When combined, their heuristics correctly identify 2,037 (86.2\%) of the 2,364 spam pages (13.8\%) in our judged collection of 17,168 pages, while misidentifying 526 spam and non-spam pages (3.1\%).

Mukherjee et al [4] implemented a Opinion on the social media such as the product reviews are now widely used by the separation and organizations for their decision making. However, due to the reason of fame, people can try to system by the opinion spamming to have promote or to demote the some target products. In recent years, fake reviews can be detection has attracted to significant attention from both the business and research field. due to the difficulty of human labeling needed for supervised learning and evaluation, the problem has to be highly challenging. This work proposes a stories as problem by modeling spamicity as latent. An unsupervised model, called Author Spamicity Model was proposed. In present literature, the most related work on finding the fake rating is focused on protocol and architecture aspect. Ranking fraud detection, evidence aggregation has not been studied so far. We will formulate a framework which solves source-address ranking fraud detection problems, depending on attack scenarios and the operator's policy and constraints.

Proceedings of the $3^{\text {rd }}$ National Conference on Image Processing, Computing, Communication, Networking and Data Analytics (NCICCNDA 2018) 
Evidence Aggregation Based Spam Detection in e-Commerce Social Network

\section{PROPOSED SYSTEM}

In this paper, we have to propose a simple algorithm to easily identify the leading sessions of each Application based on its historical ranking records. At the time with the analysis of the ranking behaviors, we have to find the fake Applications usually have to separate the ranking patterns in each leading session can differentiate with the normal Applications. So we have to identify the some fraud evidences from Applications historical ranking records, and developing the three functions to extracting the ranking based fraud evidences. And at propose two types of fraud evidences based on Applications rating and review histories, which can reflect the some anomalies patterns from Applications historical rating and review records. In Ranking Based Evidences, by analyzing the historical ranking records and we have to observe that Application ranking behaviors in a leading event always satisfy a specific ranking pattern, which consists of three different ranking phases, namely, rising phase, maintaining phase and recession phase. In Rating Based Evidences, specifically, after an Application has been published, it can be rated by any user who downloaded it. user rating is one of the most important features of Application advertisement. An Application which has higher rating may attract more users to download and can also be ranked higher in the leaderboard. Thus, rating manipulation is also an important aspect of ranking fraud. In Review Based Evidences, besides ratings, most of the Application stores also allow users to write some textual comments as Application reviews. Such reviews can reflect the personal perceptions and usage experiences of existing users for particular web Applications. Indeed, review manipulation is one of the most important aspects of Application ranking fraud.



FIG 2: Dataflow Diagram for admin part 




FIG 3: Dataflow Diagram for user part

\section{A. MAIN OBJECTIVES}

The main objective of the proposed concept is;

a. The proposed framework is scalable and can be extended with other domain generated evidences for ranking fraud detection.

b. The Experimental results show the effectiveness of the proposed system, the scalability of the detection algorithm as well as some regularity of ranking fraud activities.

c. To the best of our understanding, there is no existing benchmark to decide which leading sessions or Applications really contain ranking fraud. Thus, we will develop four intuitive baselines and invite five human evaluators to validate the effectiveness of our Approach Evidence Aggregation based Ranking Fraud Detection (EA-RFD).

Proceedings of the $3^{\text {rd }}$ National Conference on Image Processing, Computing, Communication, Networking and Data Analytics (NCICCNDA 2018) 
Evidence Aggregation Based Spam Detection in e-Commerce Social Network

\section{B. MODULES}

\section{Mining Leading Sessions}

In the first module, we will develop our system environment with the details of Application like an Application store. The leading sessions of a web Application represent its periods of popularity, so the ranking manipulation will only take place in these leading sessions. Therefore, the problem of detecting ranking fraud is to detect fraudulent leading sessions. the first task is how to mine the leading sessions of a web Application from its historical ranking records. There are two steps for mining leading sessions. First, we need to discover leading events from the Applications historical ranking records. Second, we need to merge adjacent leading events for constructing leading sessions.

\section{Ranking Based Evidences}

In this module, we will develop Ranking based Evidences system. By analyzing the Applications historical ranking records, web serve that Applications ranking behaviors in a leading event always satisfy a specific ranking pattern, which consists of three different ranking phases, as rising phase, maintaining phase and recession phase. Particularly, in each leading event, an Applications ranking is first increases to a peak position in the leader board, then keeps such peak position for a period, and finally decreases till the end of the event.

\section{Rating Based Evidences}

In the third module, we increases the system with Rating based evidence module. The ranking based evidence is useful for ranking fraud detection. Sometimes, it is not sufficient to only use ranking based evidence. For example, some Applications are created by the famous developers; they may have some leading events with large values of all due to the developers reliability and the "word-of-mouth" advertising effect.

\section{Review Based Evidences}

In this module, we adding the Review based Evidences module in our system. Apart from ratings, most of the Application stores also allow users to write some textual comments as Application reviews. Such reviews can reflect the personal perceptions and usage experiences of existing users for the particular web Applications. The review manipulation is one of the most important perspectives of Application ranking fraud. Specifically, before downloading or purchasing a new web Application, users are usually first read its historical reviews to effort their decision making, and a web Application contains more positive reviews may attract more users to download. Therefore, sometimes post fake reviews in the leading sessions of a specific Application in order to enlarged the Application downloads, and moving the Application's ranking position in the leader board.

\section{Evidence Aggregation}

In this module, we will develop the Evidence Aggregation module to our system. After extracting three types of fraud evidence, the next challenge is to combine them for ranking fraud detection. There are many ranking and evidence aggregation methods in the literature, such as permutation based models score based models and Dempster-Shafer rules. Some of 
these methods focus on learning a global ranking for all candidates. This is not proper for detecting ranking fraud for new Applications. Rather, we propose an unsupervised Approach based on fraud similarity to combine this evidence.

\section{FUTURE WORK AND CONCLUSION}

We plan to study more effective fraud evidence and analyze the latent relationship among rating, review and ranking. Moreover, we will extend our ranking fraud detection approach with other web applications related services, such as application recommendation, for enhancing user experience. In this paper, we developed a ranking fraud detection system for Social network. Specifically, we first showed that ranking fraud Application in leading sessions and provided a method for mining leading sessions for each Application from its historical ranking records. Then, we identified ranking based evidence, rating based evidence and review based evidence for detecting ranking fraud. Moreover, we proposed an optimization based aggregation method to integrate all the evidence for evaluating the credibility of leading sessions from social network Application. A unique perspective of this Application is that all the evidence can be modeled by statistical hypothesis tests, thus it is easy to be extended with other evidences from domain knowledge to detect ranking fraud. Finally, we validate the proposed system with extensive experiments on real-world web Application data.

\section{REFERENCES}

[1]. M. N. Volkovs and R. S. Zemel "A flexible generative model for preference aggregation" Copyright is held by the International World Wide Web Conference Committee (IW3C2). April 16-20, 2012, Lyon, France

[2]K. Shi and K. Ali "GetJar web Applicationlication recommendations with very sparse datasets".

[3]. A. Ntoulas, M. Najork, M. Manasse, and D. Fetterly "Detecting spam web pages through content analysis" H.5.4 [Information Interfaces and Presentation]: Hypertext/Hypermedia; K.4.m [Computers and Society]: Miscellaneous; H.4.m [Information Systems]: Miscellaneous.

[4]. A. Mukherjee, A. Kumar, B. Liu, J. Wang, M. Hsu, M. Castellanos, and R. Ghosh "Spotting opinion spammers using behavioral footprints” Copyright @ 2013 ACM 978-1-4503-2174-7/13/08...\$15.00.

[5]. A. Klementiev, D. Roth, K. Small, and I. Titov "Unsupervised rank aggregation with domain-specific expertise” Swiss NSF scholarship PBGE22-119276, and by MIAS, a DHS-IDS Center for Multimodal Information Access and Synthesis at UIUC.

[6]. Saeedreza Shehnepoor, Mostafa Salehi*, Noel Crespi "NetSpam: a Network-based Spam Detection Framework for Reviews in Online Social Media" 1556-6013 (c) 2016 IEEE. Personal use is permitted, but republication/redistributionrequiresIEEEpermission.Seehttp://www.ieee.org/publications_standards/publications/rights /index.html for more information. 